\title{
Galaxy Masses: Disks and Their Halos
}

\author{
Stacy McGaugh \\ Department of Astronomy, University of Maryland, College Park, MD 20742
}

\begin{abstract}
I review what we currently do and do not know about the masses of disk galaxies and their dark matter halos. The prognosis for disks is good: the asymptotic rotation velocity provides a good indicator of total disk mass. The prognosis for halos is bad: cuspy halos provide a poor description of the data, and the total mass of individual dark matter halos remains ill-constrained.
\end{abstract}

\section{Disk Masses}

The great regularity of the Tully-Fisher relation [32] has long been thought to originate from a strong mass-velocity relation and a near constancy of mass-tolight ratio. The latter requires a fair but not unreasonable amount of regularity in stellar populations. Put simply,円

$$
L \sim \mathcal{M} \sim V^{a} .
$$

There have long been indications (Sancisi 1995, private communication) that this simple scaling may fail at low luminosities. This has become more clear as data have improved [22, 229]. This breakdown of the Tully-Fisher relation might arise because of the chaotic star formation histories of low mass galaxies, or as a result of a breakdown in the underlying mass-velocity relation. Another possibility is that optical luminosity ceases to trace mass because stars cease to be the dominant mass component in these disks [15].

It has now become clear that this last possibility is in fact the case. Low mass galaxies are often dominated by gas rather than stars. If instead of luminosity or stellar mass, we plot disk (star + gas) mass against the flat rotation velocity, a nice mass-velocity relation is recovered over many orders of magnitude (Fig. 1). This 'Baryonic Tully-Fisher Relation' (BTF) is [21]

$$
\mathcal{M}_{d}=\mathcal{A} V_{f}^{b}
$$

for which the data in Fig. 1 give

$$
\begin{array}{r}
\mathcal{A}=50 \mathcal{M}_{\odot} \mathrm{km}^{-4} \mathrm{~s}^{4} \\
b=4.0 \pm 0.1 .
\end{array}
$$

\footnotetext{
1 "Stacy, you're a genius! ...[!] ...when it comes to pepper grinders" (van den Bosch 2001, private communication).
} 

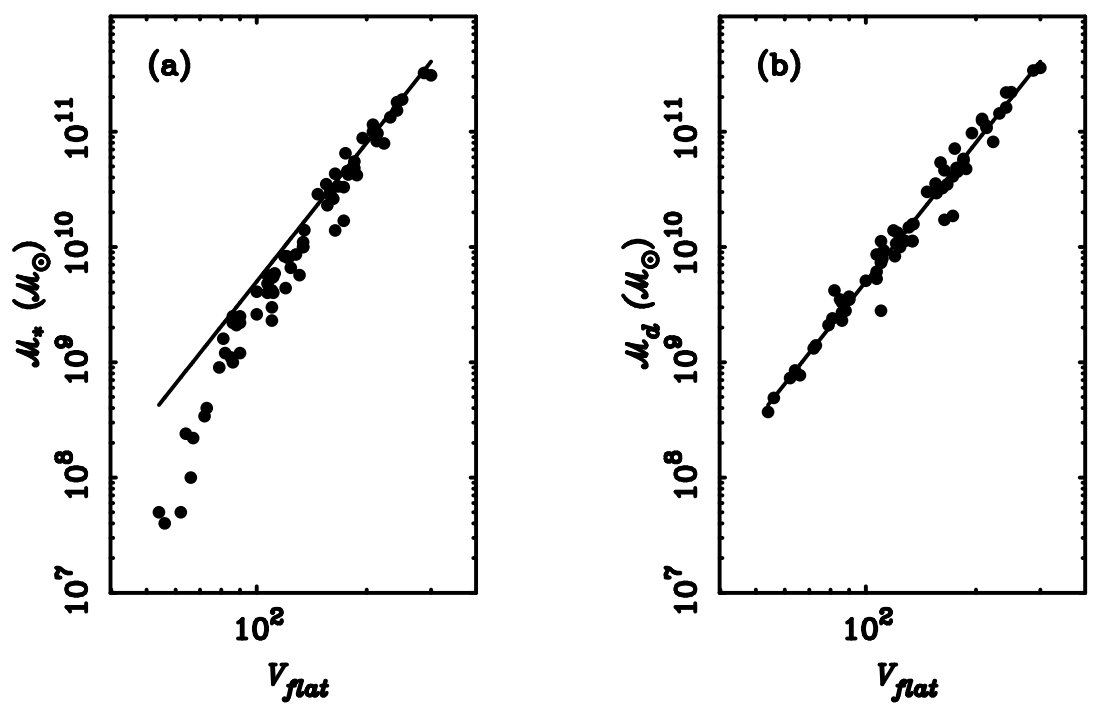

Fig. 1. The Tully-Fisher relation expressed in terms of (a) stellar mass and (b) baryonic disk mass (the sum of stars and gas). The luminous Tully-Fisher relation holds well for galaxies dominated by stars, but breaks down for low mass galaxies where the gas mass can often exceed the stellar mass (a). The sum of stars and gas provides a better correlation (b): the asymptotic flat rotation velocity is a good indicator of disk mass [21]. The data shown here are taken from a large compilation of high quality data 27]. Consequently, the scatter is greatly reduced from that in 21]. The galaxies shown here are drawn from the full range of disk Hubble types: mostly Sc, Sd, Sm, Im, but also a few $\mathrm{Sa}$ and $\mathrm{Sb}$ galaxies are present. The intrinsic scatter is small, with room only for scatter in the stellar mass-to-light ratio due to variations in star formation histories (probably not in the IMF), and scatter due to the modest ellipticities of disks [1].

The normalization of the BTF is rather uncertain: formally acceptable values fall in the range $34<\mathcal{A}<85$. The precise value of the slope has been modestly controversial: $b=4.0$ was given by [21] while $b=3.5$ was found by [2]. This difference can be traced to different assumptions about the (rather goofy [14]) distance to the UMa cluster for which some of the better rotation curve [38] and photometric data [33] exist. As the distance increases, the gas mass increases faster than the stellar mass (as $D^{2}$ and as $D$, respectively). This boosts the total mass of gas dominated galaxies by a larger factor than star dominated galaxies. Since these reside at opposite ends of the relation, the slope tips to shallower values with increasing $D$. Nevertheless, the population models of [2] are consistent with a slope of $b=4.0$ (Fig. 2). While the calibration of the BTF can always be improved, it already provides an excellent indicator of disk mass. Moreover, continuity between gas-rich and star-rich galaxies constrains stellar population mass-to-light ratios. The favored values are reasonable in terms of population synthesis models (Fig. 2), but unpleasantly heavy for cuspy dark matter halos. 

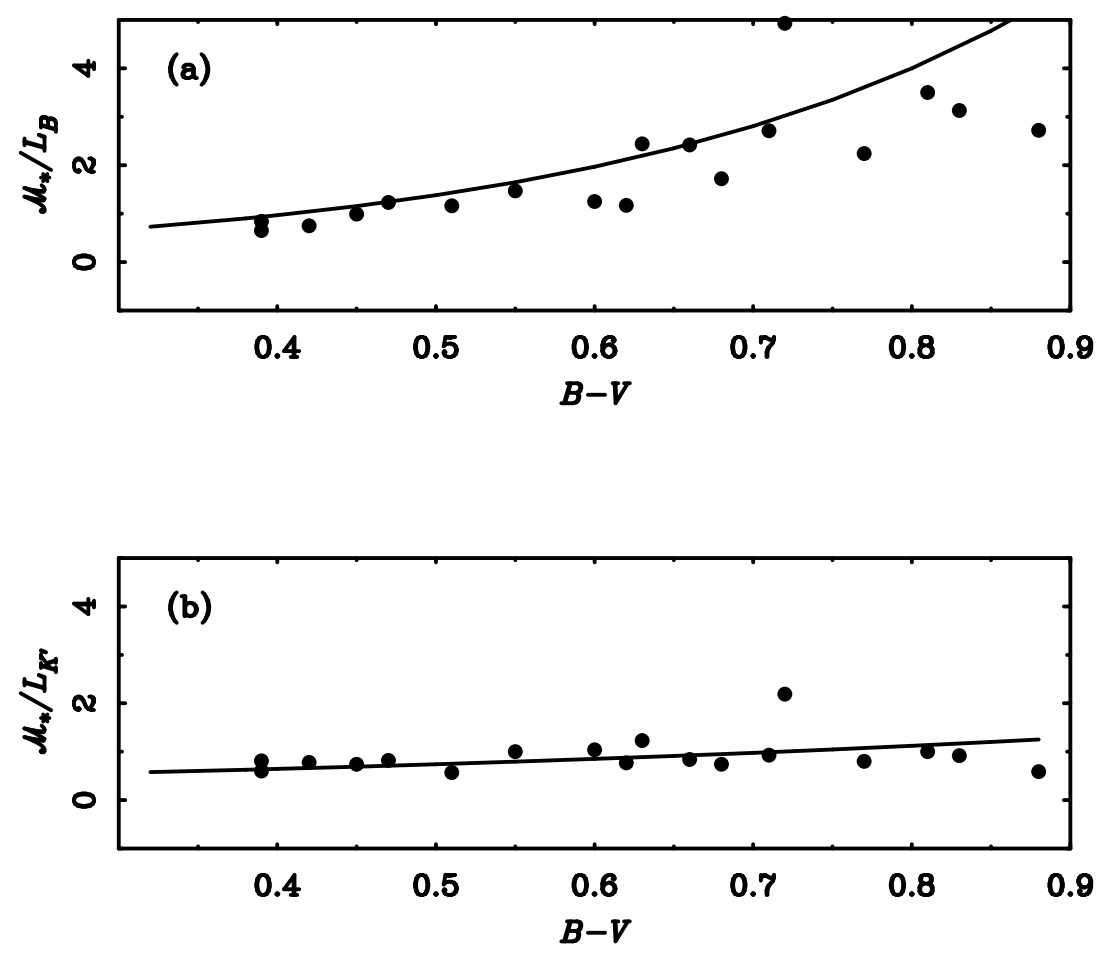

Fig. 2. The stellar mass-to-light ratios in (a) the $B$-band and (b) the $K^{\prime}$-band predicted by a slope 4 BTF for the UMa galaxies [37], [33], [38]. These are plotted as a function of $B-V$ color, together with the Bruzual \& Charlot, Salpeter IMF model from [2] (the first model in their table 4). The population synthesis models are in good agreement with the BTF, indicating that we have a good handle on $\mathcal{M}_{*} / L$ and disk masses.

\section{Dark Matter Halos}

Rotation curves, by themselves, can only give a lower limit on the total halo mass: that enclosed by the last measured point. However, if the functional form of the halo were known, it might be possible to provide some constraint by fitting the observations to the known form. The NFW halo paradigm [23], 224 which has arisen from cosmological N-body simulations in principle gives a way to do this.

Unfortunately, if not surprisingly, observed rotation curves never extend far enough to constrain the circular velocity at the virial radius, $V_{200}$ [20]. There is a great deal of degeneracy between the concentration $c$ and $V_{200}$. An example is given in Fig. 3, which shows how difficult it can be to distinguish between fits with NFW halos of rather different parameters.

Matters are made worse by the general failure of the NFW form to provide a good description of the data. The data just don't look like NFW halos. Statis- 


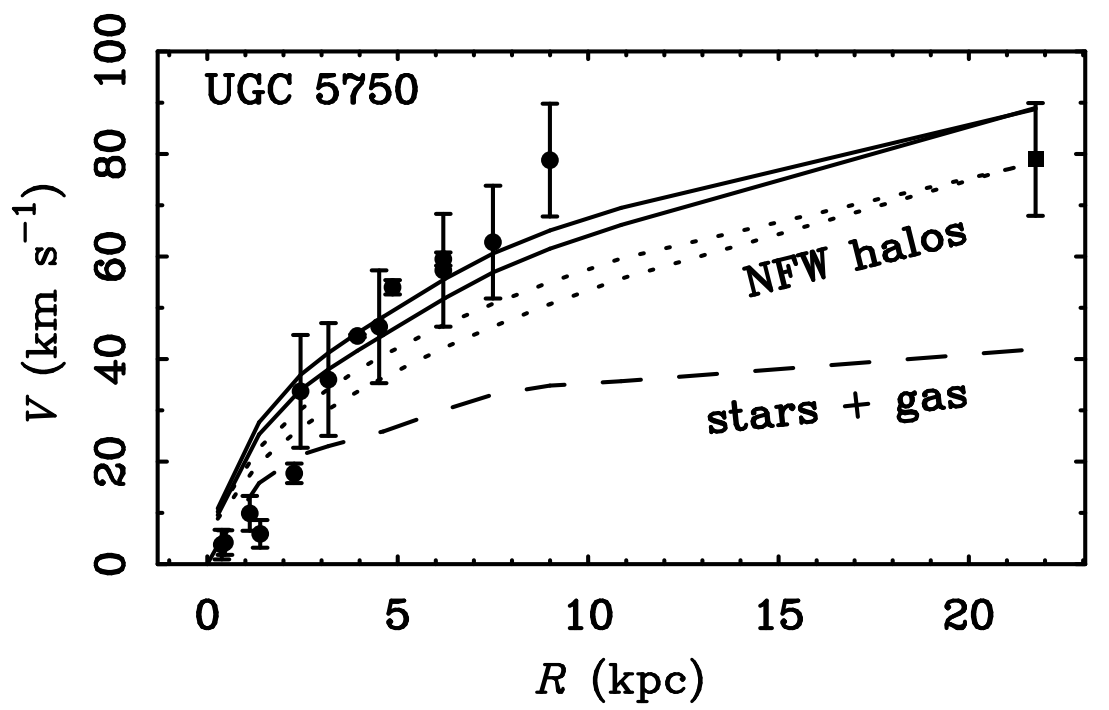

Fig. 3. An example of an NFW halo fit to the LSB galaxy UGC 5750 11. The best fit parameters in this case are $c=1.9$ and $V_{200}=117$ for $\mathcal{M}_{*} / L_{R}=1.4 \mathcal{M}_{\odot} / L_{\odot}$. Another tolerable fit with $c=0.2$ and $V_{200}=300$ is also shown (lower dotted curve) to illustrate the degeneracy between parameters. Though many models sort of fit, their concentrations are implausibly low for $\Lambda \mathrm{CDM}$.

tically, halos with constant density cores are almost always preferred over those with cusps [11]. This is most clear in the best resolved cases [10].

The most important systematic concern at this point is not observational. Resolution has improved by an order of magnitude [31], 20], [9] over the original $21 \mathrm{~cm}$ data for LSB galaxies [36], [12]. The NFW shape has not become apparent as the data have improved. Instead, the systematics pointed out by [18], 19] as problematic for CDM (independent of the cusp issue) have only become more clear. Concern over slit mispositioning [30] are misplaced: independent observers reproduce one anothers' results [20], [9]. While there are certainly cases in which the error bars are large enough to allow an NFW fit, isothermal fits are inevitably better. Simply changing the size of the error bars won't change this: a systematic change in the shapes of $\sim 50$ high resolution rotation curves is required. One can certainly imagine ways in which this might happen [30], but it is extremely unlikely that any of these ideas apply to real data, let alone to all of the data from various independent sources.

The most serious issue is in the mass models: stars have mass. Even in the limit of zero stellar mass, which is the most favorable to the NFW case, isothermal halos are statistically preferred [11], [9]. The situation only becomes more grim if stars are allowed to have mass. Though LSB galaxies are dark matter dominated down to small radii, plausible $\mathcal{M}_{*} / L$ models do require that some of 
the velocity be attributed to luminous mass. This pulls the inferred dark matter distribution further away from the expected cusp slope.

We are hardly unique in reaching these conclusions, which are shared by all published analyses of high resolution long slit $\mathrm{H} \alpha$ data [6], [8], [9], [10], [11], [13], [26]. High resolution Fabry-Perot [4, [39], [25] and CO [5] data are also inconsistent with cuspy halos, as are a variety of data for the Milky Way itself [3]. The only analyses which are favorable to NFW are those of low resolution data with large error bars [34, 335. When the error bars are large, any model can be driven through them. Though it has not been emphasized, constant density cores provide as good or better fits even in these cases.

The isothermal halo form, while effective, is an extremely flexible fitting function which lacks the motivation of the NFW halo form. So one might persists that the NFW fits are still more appropriate in that they can be related to cosmology. Standard $\Lambda \mathrm{CDM}$ makes a clear prediction 24] for what the concentrations of dark matter halos should be: $c=9$ for $\Omega_{m} h=0.2$. Scatter about this value should be modest - the largest estimate [7] finds a lognormal distribution with $\sigma_{c}=0.18$. The median observed concentration is $c=6.4$ [11] which is different from the standard $\Lambda \mathrm{CDM}$ prediction by many $\sigma$. The problem with NFW halos is not just a matter of getting fits to individual galaxies, but also of understanding how the observed concentrations can be so low. These low concentrations would be tolerable in a very low density universe with $\Omega_{m} h \approx 0.12$ [17. Even then there exists a significant tail of very low concentration $(c<4)$ galaxies which simply should not exist for any plausible cosmology.

The debate over halo profiles, while contentious, misses the real point. Many halo profiles are nominally viable because they have lots of degenerate free parameters. Mass modeling is a bit like fitting a high order polynomial to a few data points: the line goes through the data, but means nothing. One would prefer to have a minimal parameter description of the data. Such a prescription exists [16. It has long been noted that there is a strong coupling between mass and light 2 Oddly, this coupling persists for dark matter dominated LSB galaxies. One needs only a single parameter per galaxy, the stellar mass-to-light ratio, in order to fit the rotation curve in comparable or greater detail than can be matched by many-parameter halo models. The mass-to-light ratio in the $K$-band is close enough to constant that one can make a good zero parameter prediction with such data [28]. Until we come to terms with this observed phenomenology, debating the cusp slope of dark matter halos is rather akin to debating the number of angels that can dance on the head of a pin.

Acknowledgements: I am most grateful to Vera Rubin, Erwin de Blok, and Albert Bosma for their work on the issues discussed here. I would also like to thank Renzo Sancisi, Marc Verheijen, Rob Swaters, and Frank van den Bosch for many lively and stimulating conversations. No doubt, I have yet to hear the end of it! The work of SSM is supported in part by NSF grant AST9901663.

\footnotetext{
${ }^{2}$ Renzo's Rule: when you see a feature in the light, you see a corresponding feature in the rotation curve.
} 


\section{References}

1. D.R. Andersen, M.A. Bershady, L.S. Sparke, J.S. Gallagher, E.M. Wilcots: ApJ, 551, L131 (2001)

2. E.F. Bell, R.S. de Jong: ApJ 550, 212 (2001)

3. J.J. Binney, N.W. Evans: MNRAS, submitted (2002)

4. S. Blais-Ouellette, P. Amram, C. Carignan: AJ 121, 1952 (2001)

5. A.D. Bolatto, J.D. Simon, A. Leroy, L. Blitz: ApJ in press (2002)

6. A. Borriello, P. Salucci: MNRAS 323, 285 (2001)

7. J.S. Bullock, T.S. Kolatt, Y. Sigad, R.S. Somerville, A.V. Kravtsov, A.A. Klypin, J.R. Primack, A. Dekel: MNRAS 321, 559 (2001)

8. S. Côté, C. Carignan, K.C. Freeman: AJ 120, 3027 (2000)

9. W.J.G. de Blok, A. Bosma: A\&A submitted (2002)

10. W.J.G. de Blok, S.S. McGaugh, A. Bosma, V.C. Rubin: ApJ 552, L23 (2001)

11. W.J.G. de Blok, S.S. McGaugh, V.C. Rubin: AJ 122, 2396 (2001)

12. W.J.G. de Blok, S.S. McGaugh, J.M. van der Hulst: MNRAS 283, 18 (1996)

13. E. D'Onghia, these proceedings

14. W.L. Freedman et al.: ApJ 553, 47 (2001)

15. K.C. Freeman: 'Historical Introduction'. In: The Low Surface Brightness Universe, IAU Colloquium 171, ed. J.I. Davies, C. Impey, S. Phillipps (Astronomical Society of the Pacific, San Francisco 1999) pp. 3-8

16. S.S. McGaugh: 'Dynamical Constraints on Disk Galaxy Formation'. In: Galaxy Dynamics: from the Early Universe to the Present, ed. F. Combes, G.A. Mamon, V. Charmandaris (Astronomical Society of the Pacific, San Francisco 2000) pp. 153-160

17. S.S. McGaugh, M.K. Barker, W.J.G. de Blok: ApJ, submitted (2002)

18. S.S. McGaugh, W.J.G. de Blok: ApJ, 499, 41 (1998)

19. S.S. McGaugh, W.J.G. de Blok: ApJ, 499, 66 (1998)

20. S.S. McGaugh, V.C. Rubin, W.J.G. de Blok: AJ 122, 2381 (2001)

21. S.S. McGaugh, J.M. Schombert, G.D. Bothun, W.J.G. de Blok: ApJ 533, L99 (2000)

22. L.D. Matthews, W. van Driel, J.S. Gallagher 1998: AJ 116, 1169

23. B. Moore, T. Quinn, F. Governato, J. Stadel, G. Lake: MNRAS 310, 1147 (1999)

24. J.F. Navarro, C.S. Frenk, S.D.M. White: ApJ 490, 493 (1997)

25. P. Palunas, T.B. Williams: AJ 120, 2884 (2000)

26. P. Salucci: MNRAS 320, L1 (2001)

27. R.H. Sanders, S.S. McGaugh: ARA\&A in press (2002)

28. R.H. Sanders, M.A.W. Verheijen: ApJ 503, 97 (1998)

29. J.M. Stil: PhD Thesis, Leiden University (1999)

30. R.A. Swaters, these proceedings

31. R.A. Swaters, B.F. Madore, M Trewhella: ApJ 531, L107

32. R.B. Tully, J.R. Fisher: A\&A 54, 661 (1977)

33. R.B. Tully, M.A.W. Verheijen, M.J. Pierce, J. Huang, R.J. Wainscoat: AJ 112, 2471 (1996)

34. F.C. van den Bosch, B.E. Robertson, J.J. Dalcanton, W.J.G. de Blok: AJ, 119, $1579(2000)$

35. F.C. van den Bosch, R.A. Swaters: MNRAS 325, 1017 (2001)

36. J.M. van der Hulst, E.D. Skillman, T.R. Smith, G.D. Bothun, S.S. McGaugh, W.J.G. de Blok: AJ 106, 548 (1993)

37. M.A.W. Verheijen: PhD thesis, University of Groningen (1997)

38. M.A.W. Verheijen, R. Sancisi: A\&A 370, 765 (2001)

39. B.J. Weiner, J.A. Sellwood, T.B. Williams: ApJ 546, 931 (2001) 